\title{
Transdermal Solution Dosage Form
}

National Cancer Institute

\section{Source}

National Cancer Institute. Transdermal Solution Dosage Form. NCI Thesaurus. Code C149997.

Liquid single-dose or multidose preparation consisting of a solution intended for transdermal use. 